\title{
Neoadjuvant and conversion treatment of patients with colorectal liver metastasis: the potential role of bevacizumab and other antiangiogenic agents
}

\author{
Pilar García-Alfonso - Ana Ferrer • Silvia Gil • Rosario Dueñas • \\ María Teresa Pérez • Raquel Molina • Jaume Capdevila • \\ María José Safont • Carmen Castañón • Juana María Cano • \\ Ricardo Lara
}

Received: 30 November 2014 / Accepted: 28 January 2015/Published online: 11 March 2015

(C) The Author(s) 2015. This article is published with open access at Springerlink.com

\begin{abstract}
More than $50 \%$ of patients with colorectal cancer develop liver metastases. Surgical resection is the only available treatment that improves survival in patients with colorectal liver metastases (CRLM). New antiangiogenic targeted therapies, such as bevacizumab, aflibercept, and regorafenib, in combination with neoadjuvant and conversion chemotherapy may lead to improved response rates in this population of patients and increase the proportion of patients eligible for surgical resection. The present review discusses the available data for antiangiogenic targeted agents in this setting. One of these therapies, bevacizumab, which targets the vascular endothelial growth factor (VEGF) has demonstrated good results in this setting. In patients with initially unresectable CRLM, the combination of 5-fluorouracil, leucovorin, and oxaliplatin
\end{abstract}

P. García-Alfonso $(\bowtie)$

Medical Oncology Service, Hospital General Universitario Gregorio

Marañón, Madrid, Spain

e-mail: pgarcaalfonso@gmail.com

\section{A. Ferrer}

Medical Oncology Service, Hospital General Universitario de

Albacete, Albacete, Spain

S. Gil

Medical Oncology Service, Hospital Universitario Carlos Haya,

Málaga, Spain

R. Dueñas

Medical Oncology Service, Complejo Hospitalario de Jaén,

Jaén, Spain

M. T. Pérez

Medical Oncology Service, Hospital de Basurto, Bilbao, Spain

R. Molina

Medical Oncology Service, Hospital Universitario Príncipe de

Asturias, Alcalá de Henares, Madrid, Spain
(FOLFOX) plus bevacizumab has led to high response and resection rates. This combination is also effective for patients with unresectable CRLM. Moreover, the addition of bevacizumab to chemotherapy in the neoadjuvant setting of liver metastasis has a higher impact on pathological response rate. This drug also has a manageable safety profile, and according to recent data, bevacizumab may protect against the sinusoidal dilation provoked in the liver by certain cytotoxic agents. In phase II trials, antiangiogenic therapy has demonstrated benefits in the presurgical treatment of CRLM and may represent a new treatment pathway for these patients.

Keywords Antiangiogenic · Bevacizumab - Colorectal liver metastases $\cdot$ Neoadjuvant $\cdot$ Resection $\cdot$ Review

J. Capdevila

Medical Oncology Service, Hospital Universitario Vall d'Hebron,

Barcelona, Spain

M. J. Safont

Medical Oncology Service, Hospital General Universitario de

Valencia, Valencia, Spain

C. Castañón

Medical Oncology Service, Complejo Asistencial de León, León, Spain

J. M. Cano

Medical Oncology Service, Hospital General Universitario de Ciudad Real, Ciudad Real, Spain

R. Lara

Medical Oncology Service, Hospital Obispo Polanco, Teruel, Spain 


\section{Introduction}

Colorectal cancer is the second most common cause of death due to cancer in the western world. Worldwide, this disease is the third most common cause of death in women and the fourth in men [1]. There are several risk factors associated with the development of colorectal cancer such as obesity, low consumption of fruit and vegetables, a sedentary lifestyle and smoking [2-4]. These factors result in significant differences in the global incidence of the disease $[5,6]$.

Overall, more than half of patients with an initial diagnosis of colorectal cancer develop liver metastases [7]. Liver metastases at diagnosis (i.e., synchronous metastases) are present in about $25 \%$ of patients with colorectal cancer [8], and another $30 \%$ will develop them subsequently (i.e., metachronous metastases). Patients with synchronous metastases usually present poorer biological features than patients with metachronous metastases.

Surgical resection improves overall survival in patients with colorectal liver metastases (CRLM) [9]. Best patient outcomes are more frequently achieved when decisions involve a multidisciplinary team. Resectability criteria for patients with CRLM have been expanded in past years $[10,11]$. Previously, eligibility for hepatic resection was determined by the number of liver metastasis detected, the size of the tumor lesion, and the margin of resection [12]. According to recent updates, resectability criteria have been expanded to include any patient in whom all lesions can be removed with a negative margin and those who present with an appropriate liver volume or liver functional reserve [12]. Consequently, more patients are now eligible for resection by increasing or preserving liver reserve through portal vein embolization or through two-stage hepatectomy. Also, patients may become eligible with a combination of resection with ablation and decreasing tumor size by the administration of neoadjuvant and/or conversion chemotherapy. Moreover, in recent years, resectablity criteria also include patients with extrahepatic disease, as long as resection is feasible.

Although the number of patients with synchronous CRLM eligible for resection is increasing, the optimal treatment sequence in these patients is not clearly defined. Thus, surgical strategies for these patients may be described as (i) combined, i.e., resection of both primary and liver tumors; (ii) classic, i.e., resection of primary tumor before liver metastasis resection; and (iii) reverse, i.e., liver resection prior to primary tumor resection [13]. All three strategies have demonstrated efficacy in this population of patients. With regard to the long-term outcome of patients who undergo hepatic resection for CRLM, this procedure is considered to be safe and to provide good long-term survival rates, even in patients with multiple bilobar metastases [14]. Survival rates in patients undergoing a partial hepatectomy are similar to survival rates observed in patients with primary hepatic resection of CRLM [15].

In patients with unresectable CRLM, standard chemotherapy regimens which combine 5-fluorouracil with oxaliplatin or irinotecan (i.e., FOLFOX or FOLFIRI, respectively) facilitate secondary resection of liver metastases [16]. The addition of a targeted agent, for example bevacizumab, a recombinant humanized monoclonal antibody that targets the vascular endothelial growth factor receptor (VEGFR), or monoclonal antibodies that target the epidermal growth factor receptor (EGFR), may further increase the proportion of patients eligible for resection of liver metastases [17-19]. For patients with initially suboptimal or unresectable CRLM, a targeted agent plus chemotherapy also yields high response rates and leads to increased resectability [20]. In patients with initially resectable CRLM, chemotherapy improves progression-free survival (PFS) but not overall survival (OS) [21], and the further addition of an EGFR-directed antibody is not beneficial [22].

The aim of this paper is to review the role of antiangiogenic agents in the neoadjuvant treatment of patients with CRLM as well as in the conversion from initially unresectable tumors to resectable ones. In addition, the impact of these agents on pathological response, liver protection, as well as morbidity and mortality is reviewed.

\section{Methods}

A search of PubMed, ISI WoK, and Clinicaltrials.gov was performed in October 2012 for publications relating to the use of antiangiogenic agents in the neoadjuvant or conversion treatment of patients with CRLM between January 2002 and January 2012. Articles were included if published in English or Spanish. The search was divided up into six areas: (i) incidence of liver metastasis on colorectal cancer, (ii) role of surgery on liver metastasis, (iii) role of antiangiogenic drugs on liver metastasis treatment before surgery, (iv) efficacy of antiangiogenic drugs on liver metastasis of colorectal cancer, (v) clinical response and pathology response, and (vi) liver toxicity of chemotherapy (bevacizumab proctection of the liver, safety of antioangiogenics). Search terms included were as follows: antineoplastic combined chemotherapy protocols/administration and dosage; neoadjuvant therapy; neoplasm metastasis; colorectal neoplasms/drug therapy; colorectal neoplasms/pathology; colorectal neoplasms/surgery; colorectal neoplasms/therapy; liver neoplasms/drug therapy; liver neoplasms/secondary; angiogenesis inhibitors; antibodies, monoclonal; antibodies, monoclonal, humanized; and bevacizumab. Articles were reviewed by the authors for their relevance and for quality of evidence. The authors met to discuss their findings and conclusions from their review.

\section{Mechanism of action of antiangiogenic agents in colorectal cancer}

Angiogenesis is an essential process in solid tumors larger than 1-2 mm. Beyond this size, diffusion of oxygen alone is 
not enough to maintain adequate oxygen levels within the tumor tissue [23, 24]. Angiogenesis is a process stimulated by hypoxia and regulated by the balance between proangiogenic and antiangiogenic molecules. These molecules are present in endothelial cells, pericytes, and immune cells. The establishment and survival of metastases imply that a shift in the balance of these factors in favor of angiogenesis has occurred. Angiogenesis comprises several steps, including the detachment of endothelial cells and pericytes from the basement membrane, their invasion, and migration across basement membranes into the tumor mass and their differentiation to generate a new capillary network. Tumor neovascularization is a critical process in cancer progression. In fact, an increase in angiogenesis in the primary tumor has been related to poor prognosis and recurrence in colorectal cancer patients [25-28].

Vascular endothelial growth factor (VEGF) is the primary proangiogenic molecule affecting several steps throughout the angiogenic process (Table 1). The VEGF family is composed of six molecules (VEGF-A, VEGF-B, VEGF-C, VEGF-D, VEGF-E, and placental growth factor [PlGF]) and three receptors (VEGF-R1, VEGF-R2, and VEGF-R3) [29-31]. One of the VEGF-A isoforms, VEGF 165 , is commonly expressed in solid tumors [32].

Research has demonstrated that both neuropilin-1 and neuropilin-2 act as accessory receptors for the $\mathrm{VEGF}_{165}$ isoform [33], and also that overexpression of neuropilin-1 in colorectal cancer cells increases tumor growth and angiogenesis [34]. Another characteristic of neuropilin-1 is that this molecule is upregulated by the activation of EGFR, another therapeutic target for colorectal cancer.

Bevacizumab exerts its action against VEGF-A, which results in the prevention of VEGFR activation and the subsequent signaling cascades [35]. Aflibercept prevents VEGFR activation by binding to VEGF-A and PIGF [36].

Table 1 Main functions of VEGF in endothelial cells [adapted from 88]

\begin{tabular}{ll}
\hline Function & Activity \\
\hline Activation & $\begin{array}{l}\text { Upregulation of integrin expression } \\
\text { Alteration in cell cytoskeleton }\end{array}$ \\
Invasion & Induction of metalloproteinases \\
Migration & Activation of FAK and p38 \\
Permeability & Endothelial fenestrations \\
& Separation of junctions between cells \\
& Vesico-vascular organelles \\
Proliferation & Activation of MAPK \\
Survival & Induction of PI3K/PKB and survivin \\
& Inhibition of caspases \\
\hline
\end{tabular}

$F A K$ focal adhesion kinase, $M A P K$ mitogen-activated protein kinase, $P I 3 K$ phosphoinositide 3-kinase, $P K B$ protein kinase B, VEGF vascular endothelial growth factor

\section{Main antiangiogenic agents in colorectal cancer}

Three main antiangiogenic agents have been shown to improve the outcomes of patients with $\mathrm{mCRC}$ when given in combination with chemotherapy, namely, bevacizumab, aflibercept, and regorafenib.

Some of the main studies involved in the clinical development of bevacizumab are described briefly below. Hurwitz et al. conducted a phase III trial in 813 patients with previously untreated mCRC [37]. Patients were randomized to receive irinotecan plus bolus 5-fluorouracil and leucovorin (IFL) with or without bevacizumab. The addition of bevacizumab resulted in a statistical and clinical improvement in terms of median OS (20.3 vs 15.6 months, respectively; $p<0.001)$, median PFS (10.6 vs 6.2 months, respectively; $p<0.001$ ), overall response rate (RR) ( 45 vs $35 \%$, respectively; $p=0.004)$, and the median duration of the response achieved (10.4 vs 7.1 months; $p=$ 0.001 ). A significant improvement in terms of PFS ( 9.4 vs 8.0 months, respectively; $p=0.0023$ ) was also observed when bevacizumab was added to oxaliplatin-based schedules in a placebo-controlled phase III trial that included 1401 untreated patients with mCRC [38]. Moreover, results in terms of PFS were also superior in the group of patients who were treated with the addition of bevacizumab to their chemotherapy regimen until study termination [38]. Based on the data obtained in these phase III trials, together with data from other important randomized phase II trials and observational studies, bevacizumab in combination with fluoropyrimidine-based chemotherapy has been approved by the US Food and Drug Administration (FDA) and the European Medicines Agency (EMA) for the treatment of patients with mCRC.

Aflibercept is a fusion protein composed of segments of human VEGF-R1 and VEGF-R2 fused to the constant region of human immunoglobulin G1. So far, only the phase III trial VELOUR, a study carried out to evaluate the second-line treatment of patients with $\mathrm{mCRC}$ with this agent, has reported efficacy results [39]. This trial compared the addition of aflibercept or placebo to FOLFIRI in 1226 patients with $\mathrm{mCRC}$ as second-line treatment. In this setting, the addition of aflibercept improved median OS (13.5 vs 12.1 months; $p=$ 0.0032 ), median PFS (6.9 vs 4.7 months; $p<0.001$ ), and RR ( 20 vs $11 \% ; p=0.0001$ ) in patients with $\mathrm{mCRC}$ after receiving first-line treatment with oxaliplatin.

Lastly, regorafenib is a multikinase inhibitor of a wide range of angiogenic, oncogenic, and stromal kinases. The placebo-controlled randomized phase III trial CORRECT with regorafenib was conducted in 760 patients with mCRC who had progressed after receiving all available standard therapies. Median OS, the primary study endpoint, was 6.4 months in the regorafenib group versus 5.0 months in the placebo group $(p=0 \cdot 0052)[40]$.

To date, aflibercept in combination with FOLFIRI has been approved by the FDA and the EMA for the treatment of 
patients with $\mathrm{mCRC}$ who are resistant to or have progressed after an oxaliplatin-containing regimen. Regorafenib has also been approved by the FDA and the EMA in patients with $\mathrm{mCRC}$ after failure to respond to standard therapies.

\section{Main antiangiogenic agents in the neoadjuvant and conversion treatment of CRLM}

Resection of CRLM along with the administration of antineoplastic agents has become the standard of care for patients with CRLM. In this setting, bevacizumab is currently the only antiangiogenic agent that has been demonstrated, through retrospective and prospective phase II trials, to improve the outcome of patients with mCRC. Table 2 summarizes the main efficacy data from some of these trials.

\section{Retrospective studies}

Several retrospective analyses have provided evidence for the efficacy of bevacizumab when added to neoadjuvant and conversion chemotherapy for the treatment of patients with CRLM. The randomized phase III trial NO16966 compared the safety and efficacy of oxaliplatin-based chemotherapy with or without bevacizumab in 1400 patients with mCRC. A subsequent retrospective analysis of the resection rate in this study was performed [17]. In the intention-to-treat (ITT) population, 44 out of 699 (6.3\%) patients treated with chemotherapy plus bevacizumab underwent R0 metastasectomy, compared with 34 out of $701(4.9 \% ; p=0.24)$ patients treated with chemotherapy plus placebo. In the subgroup of patients with liver-only disease, curative resection rates were 12.3 and $11.6 \%$ ( $p=0.81)$, respectively.

Terrebonne et al. conducted a cohort study to assess the effectiveness of bevacizumab in combination with chemotherapy to achieve a secondary metastatic resection with curative intention in initially unresectable patients with $\mathrm{mCRC}$ [41]. Of 411 patients included, 347 were analyzable after 24 months of follow-up. Of these, $19 \%$ were able to undergo a metastatic resection of which $86 \%$ were R0-R1. Surgical patients previously received bevacizumab with either irinotecan-based regimens (97\%) or oxaliplatin-based regimens (3\%). Additionally, patients who underwent curative surgery achieved an RR of $85 \%$, which decreased to $49 \%$ in nonsurgical patients. Median PFS in surgical patients was 13.6 and 9.0 months in nonsurgical patients. Also, there were important differences in the 1- and 2-year survival rates between both populations. Additionally, for patients with initially unresectable CRLM, secondary resection was possible in $19 \%$, of whom $86 \%$ achieved a R0-R1 resection. The authors concluded that the addition of bevacizumab to chemotherapy made secondary resection possible in $19 \%$ of patients with initially unresectable mCRC.
Chaudhury et al. retrospectively reviewed 35 patients with CRLM who underwent liver resection and received bevacizumab plus chemotherapy in the perioperative period [42]. Chemotherapy consisted of an oxaliplatin-based regimen (74\% of patients), an irinotecan-based regimen (17\%), or an oxaliplatin-plus irinotecan-based regimen ( $9 \%$ of patients). The RR observed was $66 \%$. Forty-nine percent of patients underwent a portal vein embolization prior to surgery, and $34 \%$ were able to undergo staged resection for extensive bilobar disease. The 4-year survival rate was $53 \%$. These findings indicate that bevacizumab together with standard neoadjuvant chemotherapy may improve RR in these patients and may have a favorable impact on patients' survival.

Lastly, Garcia et al. conducted another retrospective analysis in 20 patients who received chemotherapy plus bevacizumab as neoadjuvant and conversion treatment. In this trial, curative resections could be performed in $95 \%$ of patients [43]. Of the resections performed, $90 \%$ were R0 ( $10 \%$ were R1). Interestingly, all synchronous primary tumors could be resected. The median disease-free survival (DFS) and OS in this series were 12.2 and 48.9 months, respectively.

\section{Prospective studies}

There are four phase II trials that have prospectively evaluated the role of bevacizumab in the perioperative treatment of patients with CRLM. The BOXER study evaluated the administration of bevacizumab with capecitabine plus oxaliplatin (XELOX) in 46 patients with CRLM who were considered unsuitable for resection due to a poor-risk prognosis [44]. Criteria for poor risk included more than four metastases, any metastases larger than 5-cm diameter, $\mathrm{R} 0$ resection unlikely, synchronous colorectal metastases, high probability of inadequate liver function in case of resection, as well as inability to retain liver vascular supply. After perioperative treatment, an RR of $78 \%$ was achieved, and 12 out of 30 patients considered unresectable before treatment were regraded to potentially resectable, which represented a conversion rate of $40 \%$. Of these patients, $20 \%$ achieved an $\mathrm{R} 0$ resection. In addition, 10 of 15 (67\%) patients with synchronous resectable CRLM underwent liver resection, and four patients underwent a "watchful waiting" approach due to an outstanding response to neoadjuvant and conversion treatment. Overall, in all eligible patients, the median PFS was 12.0 months, and the 1-year survival rate was $86 \%$.

Another nonrandomized phase II trial carried out by Gruenberger et al. included 43 patients with CRLM potentially curable by resection [45]. Patients were treated with bevacizumab plus XELOX for 5 cycles. The sixth cycle included only XELOX, leaving at least 5 weeks before surgery without bevacizumab. After treatment, $66 \%$ of patients achieved a response and $97 \%$ of patients underwent potentially curative liver resection (R0, $n=34$; R1, $n=1)$. Patients 


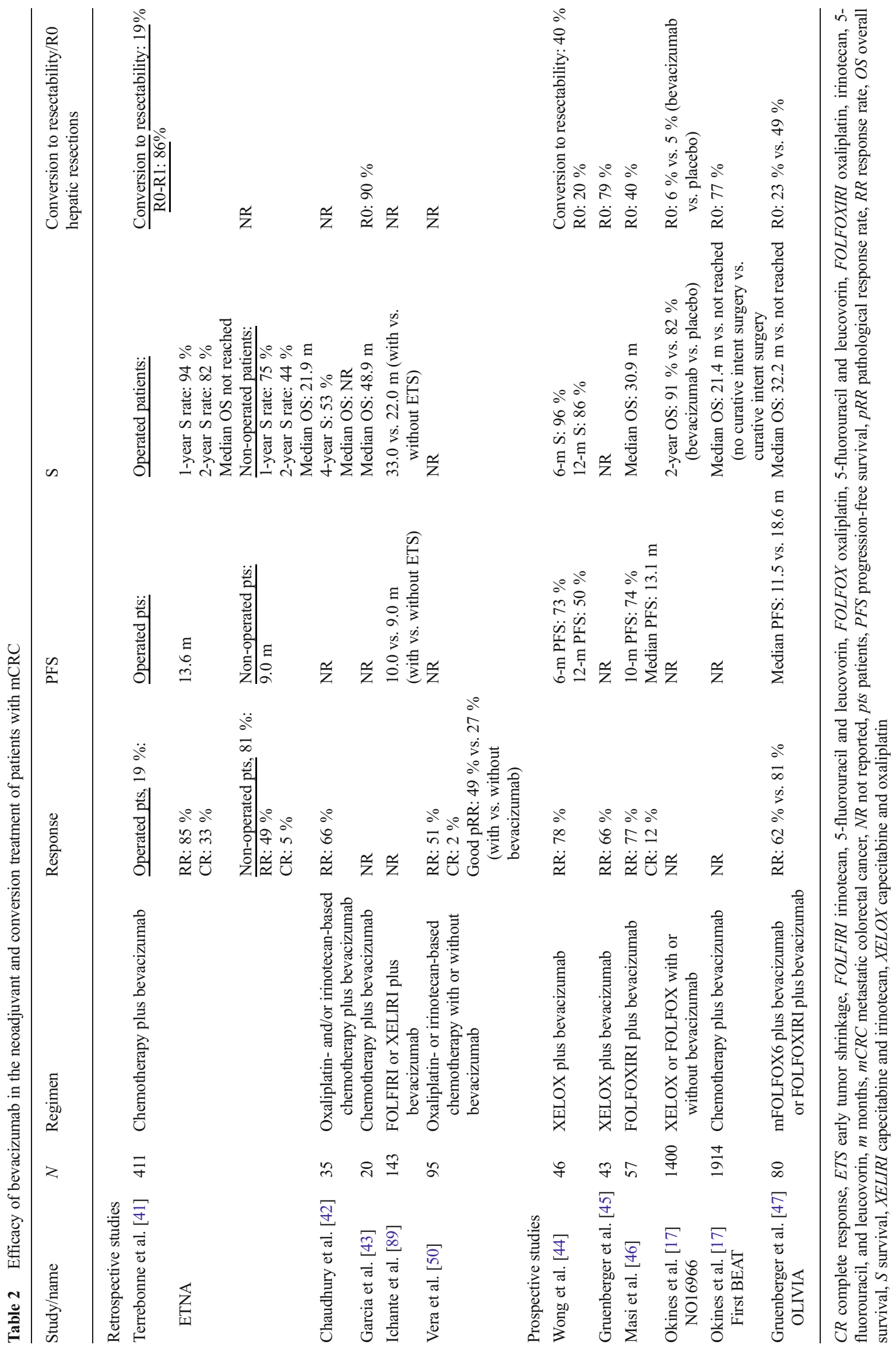


who received postoperative treatment achieved a significantly longer OS compared with patients receiving neoadjuvant treatment alone (47.9 vs 27.7 months; $p<0.05$ ).

Another prospective phase II trial assessed the safety and efficacy of the combination of bevacizumab with oxaliplatin, irinotecan, 5-fluorouracil, and leucovorin (FOLFOXIRI) in 57 patients with unresectable mCRC [46]. This combination was administered for a maximum period of 6 months followed by maintenance therapy with bevacizumab. An RR of $77 \%$ was achieved. After 10 months, the PFS rate was $74 \%$; median PFS and OS were 13.1 and 30.9 months, respectively.

Lastly, 80 patients with initially unresectable CRLM were randomized in the phase II OLIVIA trial to receive bevacizumab with modified 5-fluorouracil, leucovorin, and oxaliplatin (mFOLFOX6) or bevacizumab with FOLFOXIRI [47]. The results suggested that the combination of bevacizumab and FOLFOXIRI improved resection rates, RR and PFS compared with patients treated with bevacizumab plus mFOLFOX6. Therefore, bevacizumab with FOLFOXIRI should be further assessed in this setting.

\section{Studies evaluating pathological response}

According to recent data, a good pathological response predicts a better outcome in terms of OS in patients with advanced colorectal cancer who have undergone neoadjuvant and conversion chemotherapy prior to resection of CRLM [48-50]. Based on these results, Vera et al. conducted a retrospective assessment of 95 patients who received either irinotecan- or oxaliplatin-based chemotherapy alone or together with bevacizumab [51]. A good pathological response, i.e., complete pathological response or $<25 \%$ of residual viable tumor cells, was detected in $49 \%$ of patients receiving bevacizumab in comparison with $27 \%$ of patients receiving chemotherapy alone $(p=0.0302)$. The authors concluded that the addition of bevacizumab to chemotherapy in the neoadjuvant and conversion setting significantly improves pathological response in this population of patients.

The histological or pathological response to chemotherapy achieved in patients with CRLM may be graded according to tumor regression. Based on this relationship, resected liver metastases from patients included in two prospective nonrandomized trials were analyzed retrospectively [50]. In these trials, patients received either FOLFOX or XELOX, with or without bevacizumab. Pathological response was analyzed according to different tumor regression grades (TRGs), and these were correlated with the durations of PFS and OS observed in these patients. The authors noted that $38 \%$ of patients in the bevacizumab arm had a major histological response, in comparison with $10 \%$ of patients in the chemotherapy-only arm $(p<0.001)$. Moreover, there was a significant prolongation of both PFS and OS in patients with a major histological response compared with patients with a partial or no histological response. Thus, classifying histological response by TRGs may help to predict the outcome of patients with CRLM. In this regard, Chang et al. proposed a modified TRG (mTRG) that included two types of histological classification of tumor necrosis, namely, usual necrosis (UN), which represents a lack of treatment effect, and infarct-like necrosis (ILN), which represents a therapeutic response to chemotherapy [52]. Moreover, in a series of 109 cases, they observed that all cases of ILN were associated with perioperative treatment with chemotherapy and bevacizumab. Also, they observed that patients with ILN achieved a superior DFS compared with patients with UN $(p=0.047)$.

In another study conducted by Ribero et al. [53], results from 105 patients with CRLM treated preoperatively with 5fluorouracil plus oxaliplatin with or without bevacizumab were analyzed. It was observed that the addition of bevacizumab to chemotherapy significantly reduced the percentage of residual viable tumor cells in resected tumors compared with chemotherapy alone ( 33 vs $45 \%$, respectively; $p=$ $0.02)$. Also, after stratification according to the magnitude of tumor viability, more patients treated with bevacizumab presented with $<25 \%$ of residual viable tumor cells compared with the control arm (45 vs $23 \%$, respectively; $p=0.020$ ), although the complete pathological RR was similar in both arms ( 11 vs $12 \%$, respectively; $p=0.590$ ).

Finally, another study evaluated the association between the duration of neoadjuvant and conversion chemotherapy with FOLFOX with or without bevacizumab, the rate of pathological response and the incidence of hepatotoxicity [54]. In this study, 219 patients with CRLM underwent liver resection after a short (1-8 cycles) or long ( $\geq 9$ cycles) duration of chemotherapy with or without bevacizumab. It was observed that a long duration of treatment increased the risk of hepatotoxicity ( $42 \mathrm{vs} 26 \%$, respectively; $p=0.017$ ), but it did not affect the pathological RR ( 55 vs $57 \%$, respectively; $p=0.74$ ). It was also noted that the addition of bevacizumab was associated with a statistically significant increase in the rate of complete or major pathological responses, independent of the duration of treatment administered. Thus, the addition of a targeted therapy, such as bevacizumab, had a greater influence on pathological RR than the duration of therapy.

\section{Evaluation of response in patients with CRLM treated with antiangiogenic agents}

Since Response Evaluation Criteria in Solid Tumors (RECIST) were published in 2000, they have been widely accepted by clinicians, the pharmaceutical industry, and government authorities [55]. However, in recent years, it has been debated whether or not it would be more appropriate to move from one-dimensional assessment criteria to either volumetric or functional assessments using techniques such as positron 
emission tomography (PET) or magnetic resonance imaging (MRI). Most clinicians foresee that new cancer treatments, such as targeted therapies, will require the development and validation of new techniques to assess tumor response accurately. In line with this, several response evaluation criteria have been assessed in recent years through research in different tumor types.

In cancer patients treated with antiangiogenic agents

RECIST do not take into consideration factors such as the decrease in tumor density or the amount of intratumoral vessels which are associated with treatment with antiangiogenic agents. Nonetheless, PET has been demonstrated to have a high sensitivity for detecting early responses, as well as for predicting long-term outcomes in patients with mCRC [56]. However, the use of PET is limited by its cost. Another feasible option is computed tomography (CT), which combines an evaluation of tumor density with tumor size criteria. This technique may allow the detection of early responses, and may also have excellent prognostic value.

Smith et al. conducted a retrospective study in patients with metastatic renal cell carcinoma treated with antiangiogenic agents [57]. The standard contrast-enhanced CT (CECT) images from these patients were analyzed using four types of assessment criteria. The analysis demonstrated that the assessment was more accurate using Mass, Attenuation, Size and Structure (MASS) criteria. In another study, Wahl et al. reviewed the evaluation of tumor response according to World Health Organization, RECIST, and RECIST 1.1 criteria in patients with solid tumors [58]. The metabolic tumor response was also analyzed with F-18-fluorodeoxyglucose PET ([18]FFDG PET), and the authors suggested PET Response Criteria in Solid Tumors (PERCIST). The results showed that evaluation by anatomic imaging criteria alone has limitations, especially for new cancer therapies focused on the stabilization of disease. In contrast, (18)F-FDG PET had high value in this setting. However, further research is warranted. Lastly, another study assessed whether the tumor response achieved by patients with unresectable lung adenocarcinoma treated with chemotherapy plus an antiangiogenic agent could be evaluated by wide-volume perfusion CT [59]. The authors concluded that perfusion $\mathrm{CT}$ imaging could detect alterations in the vascularity of the tumor after treatment administration.

In patients with CRLM treated with antiangiogenic agents

Several trials have also been carried out in patients with CRLM treated with antiangiogenic agents in order to determine the optimal evaluation criteria for these patients in the neoadjuvant setting. One of these studies was conducted with the aim of evaluating new tumor response criteria based on morphological changes detected by CECT [60]. Patients selected for the study presented with a diagnosis of CRLM and were treated with bevacizumab in combination with chemotherapy in the neoadjuvant setting. CT-based morphological criteria were significantly associated with pathological response and OS. Nonetheless, morphological changes in the tumor are not always correlated with complete pathological response. Another study aimed to determine whether perfusion CT and magnetic resonance diffusion-weighted imaging have value in the assessment of response after administering chemotherapy plus an antiangiogenic agent as neoadjuvant therapy in patients with CRLM [61]. Both techniques were found to be useful for detecting therapy-induced modifications in the vascularization of metastases, which occur prior to changes in lesion size.

An assessment of the role of dynamic contrast-enhanced MRI in the evaluation of tumor response to treatment with antiangiogenic drugs was carried out in patients with CRLM [62]. This technique may be used to study the pathophysiology of tumors by measuring the microvascular density of the tumor and its vascular permeability. Results of this study showed that responders presented with a reduction in tumor perfusion within 26-33 h after the first dose of treatment. Such results suggest that dynamic contrast-enhanced MRI may be a more precise measure of pathological response to antiangiogenic agents than the determination of tumor diameter in the neoadjuvant treatment of patients with CRLM.

Lastly, the Multidisciplinary Spanish Group for Gastrointestinal Malignancies (GEMCAD) is currently performing the AVAMET study (identifier NCT01493713), which will evaluate the correlation between RR according to RECIST v1.1. evaluated by conventional imaging techniques, and morphological response by CT or histopathological response in patients with CRLM treated with XELOX plus bevacizumab. However, at the time of writing, no results are available.

\section{Prognostic criteria and response markers in patients with CRLM}

Prognostic criteria in patients with $\mathrm{mCRC}$ eligible for hepatic resection have been developed in order to ensure optimal benefit in terms of OS and PFS. These criteria have been useful because they allow patient stratification into risk categories and ensure an appropriate approach for every patient. Nevertheless, selecting the appropriate candidates for hepatic resection of CRLM has not been an easy task. Since 1980, several prognostic scoring systems have been developed [63-68]. There are obviously significant differences between them, and the general applicability of some of the scoring systems has not been clearly validated, either internally or externally, prior to their general use.

On the other hand, several promising new prognostic factors have been identified. These include the following: (i) 
markers of systemic inflammatory response, which are associated with poor outcomes [69]; (ii) microarray analysis to identify biological, histological, genetic, or proteomic "signatures"; and (iii) high-throughput screening techniques [70-73]. These new tools allow better predictive accuracy compared with current prognostic factors. Hence, in the future, prognostic scoring systems, biological and clinical factors should be tested in larger samples of patients. Nevertheless, external confirmation is also required prior to the widespread use of any new prognostic scoring system.

With regard to response markers, it is interesting to mention a study conducted in 525 patients to determine whether TRGs in patients with CRLM can predict clinical outcome in terms of DFS and OS [74]. Histological tumor regression of hepatic metastases was associated with fibrosis overgrowth, but not with an increase of necrosis. Hence, fibrosis overgrowth should be taken into account when evaluating the efficacy of chemotherapy regimens against CRLM, and histological tumor regression is associated with a better clinical outcome.

Nonetheless, in line with the presence of necrosis in the tumor, a retrospective clinical and histological review was conducted by Chang et al. in 121 partial hepatectomies performed for CRLM [52], of which 109 had appropriate data available. Among all 109 cases, 12 (11\%) presented with ILN and 95 (87 \%) showed UN. All 12 cases of ILN were associated with perioperative chemotherapy, and six of them were related to the administration of bevacizumab ( $p=0.002)$. However, no association was found between ILN and other chemotherapeutic agents. Interestingly, ILN was related to improved DFS and OS in comparison with UN. Such results strongly suggest that ILN is a form of tumor response to chemotherapy and that this type of necrosis may be a prognostic factor in this population of patients. These findings also suggest that ILN was associated with bevacizumab treatment. In other words, hypoxia from antiangiogenic treatment contributes significantly to the development of ILN.

Finally, Blazer et al. conducted a retrospective study with 305 patients who received preoperative oxaliplatin- or irinotecan-based chemotherapy to assess whether pathological response to chemotherapy was associated with survival in patients with resected CRLM [48]. Patients were treated with neoadjuvant chemotherapy followed by surgical resection, and results demonstrated that pathological response (major response $p=0.034$; hazard ratio, 4.80 ; minor response $p=$ 0.007; hazard ratio, 6.93) was an independent predictor of survival in this population of patients.

\section{The protective effect of antiangiogenic agents on hepatic injury}

The use of cytotoxic agents before resection of CRLM has been linked with liver injury, in particular preoperative irinotecan and oxaliplatin with chemotherapy-associated steatohepatitis and vascular parenchymal injury [75]. Fernandez et al. evaluated the effect of administering preoperative irinotecan and oxaliplatin on the development of steatohepatitis in 37 patients with CRLM [76]. Biopsy scores for nonalcoholic steatohepatitis were significantly worse in patients treated with irinotecan- or oxaliplatin-based chemotherapy compared with no chemotherapy or 5 -fluorouracil alone $(p=0.003)$. Moreover, steatohepatitis affected the ability to perform large liver resections. In another study, Vauthey et al. evaluated whether preoperative chemotherapy could cause hepatic injury, affecting the postoperative outcome of 406 patients with CRLM [77]. At the end of the study, it was determined that the administration of oxaliplatin was associated with sinusoidal dilation, whereas treatment with irinotecan was associated with steatohepatitis. In addition, it was found that steatohepatitis was associated with a higher 90day mortality rate after hepatic surgery. Hence, the chemotherapy regimen to be administered to this patient population should be carefully considered because the risk of hepatotoxicity can be significant.

Data regarding pathology, as well as surgical outcomes, were obtained in a study by Aloia et al. [78]. Ninety-two patients treated with 5-fluorouracil- or oxaliplatin-based regimens were randomly selected from a cohort of 303 patients who underwent CRLM resection. No differences were found in terms of steatosis regardless of whether patients had received preoperative chemotherapy or not ( $13 \mathrm{vs} 12 \%$, respectively; $p=0.86$ ). In contrast, hepatic vascular lesions were more frequent in patients who had received chemotherapy in comparison with chemotherapy-naïve patients (52 vs $18 \%$, respectively; $p=0.01$ ). Patients who had been treated preoperatively presented with a higher incidence of sinusoidal vasodilation and congestion compared with chemotherapy-naive patients ( 23 vs $12 \%$ ). The authors concluded that the main hepatic lesions induced by 5-fluorouracil- and oxaliplatinbased chemotherapy regimens are vascular lesions and not steatosis, and that the risk of postoperative complications is related to the duration of preoperative chemotherapy administration.

With the aim of assessing the effects of preoperative systemic chemotherapy on the liver, Karoui et al. [79] conducted a study in 67 patients who underwent hepatic resection for colorectal cancer. Pathological examination of the liver parenchyma demonstrated that sinusoidal dilation was present in $49 \%$ of patients who had received preoperative chemotherapy in comparison with $25 \%$ of the patients who did not receive chemotherapy in the 6 months before resection $(p=0.005)$. However, steatosis rates were similar in both groups. Ideally, the duration of treatment should be long enough to maximize response, but short enough to minimize toxicity and surgical morbidity. In line with this, Karoui et al. also observed that in the chemotherapy group, morbidity was increased among 
patients who were administered $\geq 6$ cycles of chemotherapy in comparison with patients who received $<6$ cycles ( 54 vs $19 \%$; $p=0.047$ ). Patients who did not present with adverse events (AEs) during their postoperative course had received a median of five cycles (range, 3-29) of preoperative chemotherapy. In contrast, patients with postoperative morbidity had received a median of 12 cycles (range, 6-22) of chemotherapy during this period. Hence, this study shows that prolonged systemic chemotherapy leads to pathological changes in the parts of the liver with no metastases, such as sinusoidal dilation and atrophy of hepatocytes, as well as increasing postoperative complications. In addition, the risk of postoperative morbidity is related to the number of cycles of chemotherapy administered preoperatively. Nonetheless, whether or not judiciously administered chemotherapy affects long-term outcomes is still the subject of debate and clinical investigation.

Recent data suggest that bevacizumab may protect against sinusoidal damage. With the aim of studying sinusoidal damage in patients with CRLM, Ribero et al. compared the outcomes of 62 patients treated with FOLFOX plus bevacizumab with 43 patients treated with FOLFOX alone [53]. Patients treated with bevacizumab showed a significantly lower rate of sinusoidal injury, both of any grade (27 vs $54 \% ; p<0.01$ ) and of grades $2-3$ ( 8 vs $28 \% ; p<0.01$ ). In line with this, Klinger et al. also found a statistically significantly lower rate of severe sinusoidal dilation in patients treated with FOLFOX plus bevacizumab in comparison with patients treated with FOLFOX alone ( 2 vs $24 \%$, respectively) [80]. The authors concluded that bevacizumab may protect against sinusoidal obstruction syndrome, providing a histological rationale for the safe use of bevacizumab prior to liver resection.

In addition, several studies have suggested the possibility of hepatic regeneration after bevacizumab administration. One of these studies, conducted by Aussilhou et al., found a lower incidence of hepatic hypertrophy following portal vein embolization in 13 patients treated with FOLFOX or FOLFIRI plus bevacizumab, in comparison with 27 patients treated with these chemotherapy regimens without bevacizumab [81]. In addition, patients treated with a longer duration of chemotherapy plus bevacizumab presented with substantially less hepatic hypertrophy after undergoing portal vein embolization than patients treated with shorter schedules.

\section{Safety profile of antiangiogenic agents}

Angiogenesis is a complex process where VEGF plays an important role in multiple biological pathways, such as hematopoiesis, myelopoiesis, and endothelial cell survival. Because of this, antiangiogenic therapy shows a different toxicity profile than standard chemotherapy regimens. However, bevacizumab, the most established antiangiogenic agent, does not substantially alter the toxicity profile of chemotherapy in patients with mCRC [82]. Additionally, bevacizumabassociated hematological or gastrointestinal toxicities do not overlap with the toxicities of chemotherapy agents. In the BEAT study [83], in which bevacizumab was combined with FOLFOX, XELOX, FOLFIRI, or fluoropyrimidines alone, the main severe AEs associated with bevacizumab occurred in $11 \%$ of patients and included bleeding (3\%), gastrointestinal perforation (2\%), arterial thromboembolism (1\%), hypertension ( $5 \%$ ), proteinuria ( $1 \%$ ), and wound-healing complications $(1 \%)$, and the rates were similar regardless of the chemotherapy schedule administered. Bleeding events were generally mild in resected and unresected patients, and gastrointestinal perforation was observed in $4 \%$ of patients with unresected primary tumors. Moreover, perforation occurred at the tumor site in three patients. The 60-day mortality was $3 \%$ in the ITT study population.

To evaluate perioperative complications associated with bevacizumab, a retrospective study was carried out in 35 patients with $\mathrm{mCRC}$ who received chemotherapy plus bevacizumab. At the institution where the study was performed, a break of 6-8 weeks between the last dose of bevacizumab and liver resection was suggested [42]. Of the 35 patients included in the study, 15 patients developed a total of 23 complications after surgery, but only 5 of them $(22 \%)$ were grade III or higher according to the Clavien system [84]. Ten patients experienced 13 infectious events, 2 patients showed bile leaks, 1 patient presented with a perioperative myocardial infarction, and another experienced anastomotic dehiscence which required further surgery. Bowel obstruction was detected in one patient, and two patients developed severe liver dysfunction. One of these patients developed grade III hepatic encephalopathy after a second resection. Overall, the incidence of postoperative morbidity was high (42\%), but there was no perioperative mortality.

Since it has been suggested that bevacizumab, due to its antiangiogenic properties, can interfere with wound healing, which is relevant in the perioperative setting [85, 86], some preventive measures can be recommended in order to avoid surgical morbidity. One recommendation is that bevacizumab treatment should be stopped at least 5-8 weeks before surgery and should not be restarted until 28 days after surgery or when all incisions have healed completely [85-87]. However, shorter timelines than these may be possible. Gruenberger et al. [45] observed that in patients who had received neoadjuvant chemotherapy plus bevacizumab, and bevacizumab was discontinued about 5 weeks before surgery, surgical complication rates were similar to those observed in patients treated with chemotherapy alone. The authors concluded that it is not yet possible to determine if stopping bevacizumab for several weeks before surgery is preferable to using the agent to enable patients to undergo a complete resection. Overall, these studies demonstrated that bevacizumab is feasible in the perioperative setting, with marked effect on surgical complication rates. 
Aflibercept has demonstrated a similar toxicity profile to other agents targeting the VEGF pathway. However, aflibercept seems to increase chemotherapy-specific complications such as diarrhea, asthenic conditions, neutropenia, and stomatitis/ulceration. In the previously mentioned VELOUR trial [39], the most common AEs were anemia, diarrhea, neutropenia, asthenia, and proteinuria. Hypertension was readily managed with standard antihypertensives such as angiotensinconverting enzyme inhibitors, calcium channel blockers, and diuretics. Gastrointestinal perforations and fistulae occurred in less than $2 \%$ of patients. However, AEs leading to treatment discontinuation occurred in 27 and $12 \%$ of patients in aflibercept and placebo groups, respectively. The most common causes for treatment discontinuation in patients treated with aflibercept were asthenic conditions, infections, diarrhea, and hypertension.

The most common severe AEs found with the administration of regorafenib in the phase III CORRECT trial [40] included hand-foot skin syndrome (17\%), fatigue (10\%), diarrhea (7\%), hypertension (7\%), and rash or desquamation (6\%). No unexpected AEs were detected in patients treated with regorafenib. Nevertheless, data are scarce regarding the use of aflibercept and regorafenib with hepatic resection.

In Table 3, reported grade 3/4 AEs in phase III trials of bevacizumab, aflibercept, and regorafenib in the treatment of patients with mCRC is summarized. However, it is important to highlight that data are not really comparable as they are obtained from different clinical trials and different patient populations.

\section{Conclusions}

Perioperative treatment of patients with CRLM results in longer PFS and OS. In addition, in patients with high-risk features, neoadjuvant and conversion treatment provides an opportunity to gather information on the biological activity of the tumor and its response to treatment, as well as improving resectability rates.

To date, three antiangiogenic agents have been demonstrated to improve the outcomes of patients with $\mathrm{mCRC}$ in prospective randomized trials. Aflibercept in combination with FOLFIRI as second-line treatment improves OS and PFS in patients with $\mathrm{mCRC}$ after first-line treatment with an oxaliplatin-based regimen, with or without an antiangiogenic agent. Regorafenib has also demonstrated promising results in patients with mCRC who had progressed after receiving all approved standard treatments. However, bevacizumab is currently the only antiangiogenic drug which has demonstrated benefits in the presurgical treatment of CRLM as well as in patients with mCRC.

For patients with initially unresectable CRLM, chemotherapy with or without a targeted agent may downsize metastases and facilitate secondary resection. For these patients, the

Table 3 Reported grade 3/4 AEs in phase III trials of main antiangiogenic agents for the treatment of patients with mCRC

\begin{tabular}{|c|c|c|c|c|}
\hline Regimen & $\begin{array}{l}\text { IFL+bevacizumab, } \\
N=393 \text { [37] }\end{array}$ & $\begin{array}{l}\text { FOLFOX-4 or XELOX }+ \\
\text { bevacizumab, } N=694 \text { [38] }\end{array}$ & $\begin{array}{l}\text { FOLFIRI+aflibercept, } \\
N=611 \text { [39] }\end{array}$ & $\begin{array}{l}\text { BSC+regorafenib, } \\
N=500[40]\end{array}$ \\
\hline \multicolumn{5}{|l|}{ Hematologic toxicity, $n(\%)$} \\
\hline Leukopenia & $145(37)$ & & & \\
\hline Neutropenia & & & $-(37)$ & \\
\hline Proteinuria & $3(1)$ & $4(1)$ & $-(8)$ & $7(1)$ \\
\hline \multicolumn{5}{|l|}{ Nonhematologic toxicity, $n(\%)$} \\
\hline Arterial thrombosis & & $12(2)$ & $-(2)$ & \\
\hline Bleeding & $12(3)$ & $13(2)$ & $-(3)$ & \\
\hline Diarrhea & $126(32)$ & & $-(19)$ & $36(7)$ \\
\hline Fatigue & & & & $48(10)$ \\
\hline Fistula/intra-abdominal abscess & & $6(1)$ & $-(1)$ & \\
\hline Gastrointestinal perforation & $6(2)$ & $4(1)$ & $-(1)$ & \\
\hline Hand-foot skin reaction & & & $-(3)$ & $83(17)$ \\
\hline Hyperbilirubinemia & & & & $40(8)$ \\
\hline Hypertension & $43(11)$ & $26(4)$ & $-(19)$ & $36(7)$ \\
\hline Thrombophlebitis & $35(9)$ & & & \\
\hline Venous thrombosis & & $54(8)$ & $-(8)$ & \\
\hline Wound healing complications & & $1(1)$ & & \\
\hline Any grade $3 / 4$ toxicity, $n(\%)$ & $334(85)$ & $555(80)$ & $-(83)$ & $270(54)$ \\
\hline
\end{tabular}

$A E$ adverse event, $B S C$ best supportive care, $m C R C$ metastatic colorectal cancer, IFL irinotecan plus bolus 5-fluorouracil and leucovorin, FOLFIRI irinotecan plus infusional 5-fluorouracil and leucovorin, FOLFOX 5-fluorouracil, leucovorin, and oxaliplatin, XELOX capecitabine and oxaliplatin 
combination of FOLFOX or FOLFOXIRI plus bevacizumab has led to high RRs and resection rates, as well as an acceptable safety profile. FOLFOX plus bevacizumab is also effective for patients with unresectable CRLM and has a favorable safety profile, whereas irinotecan carries a higher risk of hepatic toxicity.

Encouraging results regarding the impact of bevacizumab plus chemotherapy on pathological response have been reported, with a clear benefit on OS. Additionally, several studies have shown that bevacizumab may have a protective effect against liver injury, in particular steatohepatitis and vascular parenchymal injuries, which are associated with the preoperative administration of irinotecan and oxaliplatin. Regarding the safety profile of bevacizumab, in order to avoid postoperative morbidities, it has been recommended that bevacizumab administration should not be restarted until 28 days after surgery or when all incisions have healed.

Antiangiogenic agents represent an increasingly important treatment option in the neoadjuvant and conversion therapy of CRLM.

Acknowledgments The authors wish to thank Ana Martín from HealthCo SL (Madrid, Spain) for her help in preparing the first draft of this manuscript. The necessary scientific meetings along with medical writing services were supported financially by Roche Farma, S.A. of Spain. Roche Farma, S.A. of Spain was given the opportunity to comment on the first draft of the manuscript, but all the decisions about its content were taken by the authors. All authors have approved the final version of the submitted manuscript.

Conflict of interest Author reports personal fees from Roche, during the conduct of the study. Author reports nonfinancial support from Roche, during the conduct of the study; personal fees from Roche, personal fees from Amgen, personal fees from Merck, outside the submitted work. Author reports personal fees from Roche, during the conduct of the study. The authors declare that they have no conflict of interest.

Open Access This article is distributed under the terms of the Creative Commons Attribution Noncommercial License which permits any noncommercial use, distribution, and reproduction in any medium, provided the original author(s) and the source are credited.

\section{References}

1. Parkin DM, Bray F, Ferlay J, Pisani P (2005) Global cancer statistics, 2002. CA Cancer J Clin 55:74-108

2. Botteri E, Iodice S, Bagnardi V, Raimondi S, Lowenfels AB, Maisonneuve P (2008) Smoking and colorectal cancer. JAMA 300: 2765-2778

3. Giovannucci E (2002) Modifiable risk factors for colon cancer. Gastroenterol Clin North Am 31:925-943

4. Giovannucci E, Wu K (2006) Cancer Epidemiology and Prevention. In: Schottenfeld D, Fraumeni J (eds) Cancers of the colon and rectum, 3rd edn. Oxford University Press, New York

5. Center MM, Jemal A, Ward E (2009) International trends in colorectal cancer incidence rates. Cancer Epidemiol Biomarkers Prev 18: $1688-1694$

6. Parkin DM (2004) International variation. Oncogene 23:6329-6340
7. Lordan JT, Karanjia ND, Quiney N, Fawcett WJ, Worthington TR (2009) A 10-year study of outcome following hepatic resection for colorectal liver metastases - The effect of evaluation in a multidisciplinary team setting. Eur J Surg Oncol 35:302-306

8. van der Pool AE, Lalmahomed ZS, Ozbay Y, de Wilt JH, Eggermont AM, Jzermans JN et al (2010) 'Staged' liver resection in synchronous and metachronous colorectal hepatic metastases: differences in clinicopathological features and outcome. Colorectal Dis 12(10 Online): e229-235

9. Artigas V, Marin-Hargreaves G, Marcuello E, Pey A, Gonzalez JA, Rodriguez M et al (2007) Surgical resection of liver metastases from colorectal carcinoma. Experience in Sant Pau Hospital. Cir Esp 81: 339-344

10. de Haas RJ, Wicherts DA, Andreani P, Pascal G, Saliba F, Ichai P et al (2011) Impact of expanding criteria for resectability of colorectal metastases on short- and long-term outcomes after hepatic resection. Ann Surg 253:1069-1079

11. Maroulis I, Karavias DD, Karavias D (2011) General principles of hepatectomy in colorectal liver metastases. Tech Coloproctol 15(Suppl 1):S13-16

12. Pawlik TM, Schulick RD, Choti MA (2008) Expanding criteria for resectability of colorectal liver metastases. Oncologist 13:51-64

13. Brouquet A, Mortenson MM, Vauthey JN, Rodriguez-Bigas MA, Overman MJ, Chang GJ et al (2010) Surgical strategies for synchronous colorectal liver metastases in 156 consecutive patients: classic, combined or reverse strategy? J Am Coll Surg 210(6):934-941

14. Wei AC, Greig PD, Grant D, Taylor B, Langer B, Gallinger S (2006) Survival after hepatic resection for colorectal metastases: a 10-year experience. Ann Surg Oncol 13:668-676

15. van der Pool AE, Lalmahomed ZS, de Wilt JH, Eggermont AM, Ijzermans JM, Verhoef C (2009) Local treatment for recurrent colorectal hepatic metastases after partial hepatectomy. J Gastrointest Surg 13:890-895

16. Adam R, Delvart V, Pascal G, Valeanu A, Castaing D, Azoulay D, Giacchetti S, Paule B, Kunstlinger F, Ghémard O, Levi F, Bismuth H (2004) Rescue surgery for unresectable colorectal liver metastases downstaged by chemotherapy: a model to predict long-term survival. Ann Surg 240:644-57

17. Okines A, Puerto OD, Cunningham D, Chau I, Van Cutsem E, Saltz L, Cassidy J (2009) Surgery with curative-intent in patients treated with first-line chemotherapy plus bevacizumab for metastatic colorectal cancer First BEAT and the randomised phase-III NO16966 trial. Br J Cancer 101:1033-8

18. Bokemeyer C, Bondarenko I, Makhson A, Hartmann JT, Aparicio J, de Braud F et al (2009) Fluorouracil, leucovorin, and oxaliplatin with and without cetuximab in the first-line treatment of metastatic colorectal cancer. J Clin Oncol 27:663-671

19. Douillard JY, Siena S, Cassidy J, Tabernero J, Burkes R, Barugel M et al (2014) Final results from PRIME: randomized phase III study of panitumumab with FOLFOX4 for first-line treatment of metastatic colorectal cancer. Ann Oncol 25:1346-55

20. Folprecht G, Gruenberger T, Bechstein WO, Raab HR, Lordick F, Hartmann JT et al (2010) Tumour response and secondary resectability of colorectal liver metastases following neoadjuvant chemotherapy with cetuximab: the CELIM randomised phase 2 trial. Lancet Oncol 11:38-47

21. Nordlinger B, Sorbye H, Glimelius B, Poston GJ, Schlag PM, Rougier P et al (2013) Perioperative FOLFOX4 chemotherapy and surgery versus surgery alone for resectable liver metastases from colorectal cancer (EORTC 40983): long-term results of a randomised, controlled, phase 3 trial. Lancet Oncol 14: $1208-15$

22. Primrose J, Falk S, Finch-Jones M, Valle J, O'Reilly D, Siriwardena A et al (2014) Systemic chemotherapy with or without cetuximab in patients with resectable colorectal liver metastasis: the New EPOC randomised controlled trial. Lancet Oncol 15:601-11 
23. Ezekowitz RA, Mulliken JB, Folkman J (1992) Interferon alfa-2a therapy for life-threatening hemangiomas of infancy. N Engl J Med 326:1456-1463

24. Ferrara N (2000) Vascular endothelial growth factor and the regulation of angiogenesis. Recent Prog Horm Res 55:15-35, discussion 35-16

25. Takahashi Y, Kitadai Y, Bucana CD, Cleary KR, Ellis LM (1995) Expression of vascular endothelial growth factor and its receptor, KDR, correlates with vascularity, metastasis, and proliferation of human colon cancer. Cancer Res 55:3964-3968

26. Takahashi Y, Tucker SL, Kitadai Y, Koura AN, Bucana CD, Cleary KR et al (1997) Vessel counts and expression of vascular endothelial growth factor as prognostic factors in node-negative colon cancer. Arch Surg 132:541-546

27. Takebayashi Y, Aklyama S, Yamada K, Akiba S, Aikou T (1996) Angiogenesis as an unfavorable prognostic factor in human colorectal carcinoma. Cancer 78:226-231

28. Vermeulen PB, Van den Eynden GG, Huget P, Goovaerts G, Weyler $J$, Lardon F et al (1999) Prospective study of intratumoral microvessel density, p53 expression and survival in colorectal cancer. Br J Cancer 79:316-322

29. Gagnon ML, Bielenberg DR, Gechtman Z, Miao HQ, Takashima S, Soker $S$ et al (2000) Identification of a natural soluble neuropilin-1 that binds vascular endothelial growth factor: In vivo expression and antitumor activity. Proc Natl Acad Sci U S A 97:2573-2578

30. Matsumoto T, Claesson-Welsh L (2001) VEGF receptor signal transduction. Sci STKE 112:re21

31. Stoeltzing O, Liu W, Reinmuth N, Parikh A, Ahmad SA, Jung YD et al (2003) Angiogenesis and antiangiogenic therapy of colon cancer liver metastasis. Ann Surg Oncol 10:722-733

32. Stimpfl M, Tong D, Fasching B, Schuster E, Obermair A, Leodolter S et al (2002) Vascular endothelial growth factor splice variants and their prognostic value in breast and ovarian cancer. Clin Cancer Res 8:2253-2259

33. Soker S, Takashima S, Miao HQ, Neufeld G, Klagsbrun M (1998) Neuropilin-1 is expressed by endothelial and tumor cells as an isoform-specific receptor for vascular endothelial growth factor. Cell 92:735-745

34. Parikh AA, Fan F, Liu WB, Ahmad SA, Stoeltzing O, Reinmuth N et al (2004) Neuropilin-1 in human colon cancer: expression, regulation, and role in induction of angiogenesis. Am J Pathol 164:2139 2151

35. Kim KJ, Li B, Winer J, Armanini M, Gillett N, Phillips HS et al (1993) Inhibition of vascular endothelial growth factor-induced angiogenesis suppresses tumour growth in vivo. Nature 362:841-844

36. Holash J, Davis S, Papadopoulos N, Croll SD, Ho L, Russell M et al (2002) VEGF-Trap: a VEGF blocker with potent antitumor effects. Proc Natl Acad Sci U S A 99:11393-11398

37. Hurwitz H, Fehrenbacher L, Novotny W, Cartwright T, Hainsworth J, Heim W et al (2004) Bevacizumab plus irinotecan, fluorouracil, and leucovorin for metastatic colorectal cancer. N Engl J Med 350:23352342

38. Saltz LB, Clarke S, Diaz-Rubio E, Scheithauer W, Figer A, Wong R et al (2008) Bevacizumab in combination with oxaliplatin-based chemotherapy as first-line therapy in metastatic colorectal cancer: a randomized phase III study. J Clin Oncol 26:2013-2019

39. Van Cutsem E, Tabernero J, Lakomy R, Prenen H, Prausová J, Macarulla T et al (2012) Addition of aflibercept to fluorouracil, leucovorin, and irinotecan improves survival in a phase III randomized trial in patients with metastatic colorectal cancer previously treated with an oxaliplatin-based regimen. J Clin Oncol 30:34993506

40. Grothey A, Van Cutsem E, Sobrero A, Siena S, Falcone A, Ychou M et al (2013) Regorafenib monotherapy for previously treated metastatic colorectal cancer (CORRECT): an international, multicentre, randomised, placebo-controlled, phase 3 trial. Lancet 381:303-312
41. Terrebonne E, Smith D, Becouarn Y, Michel P, Guimbaud R, Auby D et al (2010) Resection of colorectal cancer (CRC) metastases after bevacizumab (BV) treatment for first-line therapy: Results of the ETNA cohort study. J Clin Oncol 28(15 suppl):3594

42. Chaudhury P, Hassanain M, Bouganim N, Salman A, Kavan P, Metrakos P (2010) Perioperative chemotherapy with bevacizumab and liver resection for colorectal cancer liver metastasis. HPB (Oxford) 12:37-42

43. Garcia P, Alvarez S, Munoz A, Lopez P, Riesco M, Adeva Alfonso J et al (2011) Neoadjuvant bevacizumab treatment for colorectal liver metastasis: A retrospective study. J Clin Oncol 29(4 suppl):613

44. Wong R, Cunningham D, Barbachano Y, Saffery C, Valle J, Hickish $T$ et al (2011) A multicentre study of capecitabine, oxaliplatin plus bevacizumab as perioperative treatment of patients with poor-risk colorectal liver-only metastases not selected for upfront resection. Ann Oncol 22:2042-2048

45. Gruenberger T, Längle F, Thaler J, Eisterer W, Lenauer A, Stremitzer $S$ et al (2012) Perioperative chemotherapy including bevacizumab in potentially curable metastatic colorectal cancer; a multicenter, single arm phase I/II trial: ASSO LM1. Ann Oncol 23(suppl 4):iv19-30, PD-0004

46. Masi G, Loupakis F, Salvatore L, Fornaro L, Cremolini C, Cupini S et al (2010) Bevacizumab with FOLFOXIRI (irinotecan, oxaliplatin, fluorouracil, and folinate) as first-line treatment for metastatic colorectal cancer: a phase 2 trial. Lancet Oncol 11:845-852

47. Gruenberger T, Bridgewater J, Chau I, García Alfonso P, Rivoire M, Mudan S, et al. (2014) Bevacizumab plus mFOLFOX-6 or FOLFOXIRI in patients with initially unresectable liver metastases from colorectal cancer: the OLIVIA multinational randomised phase II trial. Ann Oncol 2014 Dec 23. pii: mdu580. [Epub ahead of print]

48. Blazer DG 3rd, Kishi Y, Maru DM, Kopetz S, Chun YS, Overman MJ et al (2008) Pathologic response to preoperative chemotherapy: a new outcome end point after resection of hepatic colorectal metastases. J Clin Oncol 26:5344-5351

49. Gruenberger T, Arnold D, Rubbia-Brandt L (2012) Pathologic response to bevacizumab-containing chemotherapy in patients with colorectal liver metastases and its correlation with survival. Surg Oncol 21:309-315

50. Klinger M, Tamandl D, Eipeldauer S, Hacker S, Herberger B, Kaczirek K et al (2010) Bevacizumab improves pathological response of colorectal cancer liver metastases treated with XELOX/ FOLFOX. Ann Surg Oncol 17:2059-2065

51. Vera R, Figueras J, Gomez Dorronsoro M, Lopez Ben S, Viudez A, Queralt B et al (2012) Retrospective analysis of pathological response in colorectal cancer liver metastases following treatment with bevacizumab: Updated findings. J Clin Oncol 30(15_suppl):e14120

52. Chang HH, Leeper WR, Chan G, Quan D, Driman DK (2012) Infarct-like necrosis: a distinct form of necrosis seen in colorectal carcinoma liver metastases treated with perioperative chemotherapy. Am J Surg Pathol 36:570-576

53. Ribero D, Wang H, Donadon M, Zorzi D, Thomas MB, Eng C et al (2007) Bevacizumab improves pathologic response and protects against hepatic injury in patients treated with oxaliplatin-based chemotherapy for colorectal liver metastases. Cancer 110:2761-2767

54. Kishi Y, Zorzi D, Contreras CM, Maru DM, Kopetz S, Ribero D et al (2010) Extended preoperative chemotherapy does not improve pathologic response and increases postoperative liver insufficiency after hepatic resection for colorectal liver metastases. Ann Surg Oncol 17: 2870-2876

55. Eisenhauer EA, Therasse P, Bogaerts J, Schwartz LH, Sargent D, Ford R et al (2009) New response evaluation criteria in solid tumours: revised RECIST guideline (version 1.1). Eur J Cancer 45:228-247

56. Lastoria S, Piccirillo MC, Caracò C, Nasti G, Aloj L, Arrichiello C, de Lutio di Castelguidone E, Tatangelo F, Ottaiano A, Iaffaioli RV, Izzo F, Romano G, Giordano P, Signoriello S, Gallo C, Perrone F (2013) Early PET/CT scan is more effective than RECIST in 
predicting outcome of patients with liver metastases from colorectal cancer treated with preoperative chemotherapy plus bevacizumab. J Nucl Med 54:2062-2069

57. Smith AD, Shah SN, Rini BI, Lieber ML, Remer EM (2010) Morphology, Attenuation, Size, and Structure (MASS) criteria: assessing response and predicting clinical outcome in metastatic renal cell carcinoma on antiangiogenic targeted therapy. AJR Am J Roentgenol 194:1470-1478

58. Wahl RL, Jacene H, Kasamon Y, Lodge MA (2009) From RECIST to PERCIST: Evolving considerations for PET response criteria in solid tumors. J Nucl Med 50(Suppl 1):122S-150S

59. Fraioli F, Anzidei M, Zaccagna F, Mennini ML, Serra G, Gori B et al (2011) Whole-tumor perfusion CT in patients with advanced lung adenocarcinoma treated with conventional and antiangiogenetic chemotherapy: initial experience. Radiology 259:574-582

60. Chun YS, Vauthey JN, Boonsirikamchai P, Maru DM, Kopetz S, Palavecino $\mathrm{M}$ et al (2009) Association of computed tomography morphologic criteria with pathologic response and survival in patients treated with bevacizumab for colorectal liver metastases. JAMA 302:2338-2344

61. Anzidei M, Napoli A, Zaccagna F, Cartocci G, Saba L, Menichini G et al (2011) Liver metastases from colorectal cancer treated with conventional and antiangiogenetic chemotherapy: evaluation with liver computed tomography perfusion and magnetic resonance diffusion-weighted imaging. J Comput Assist Tomogr 35:690-696

62. Morgan B, Thomas AL, Drevs J, Hennig J, Buchert M, Jivan A et al (2003) Dynamic contrast-enhanced magnetic resonance imaging as a biomarker for the pharmacological response of PTK787/ZK 222584, an inhibitor of the vascular endothelial growth factor receptor tyrosine kinases, in patients with advanced colorectal cancer and liver metastases: results from two phase I studies. J Clin Oncol 21:3955-3964

63. Fong Y, Fortner J, Sun RL, Brennan MF, Blumgart LH (1999) Clinical score for predicting recurrence after hepatic resection for metastatic colorectal cancer: analysis of 1001 consecutive cases. Ann Surg 230:309-318, discussion 318-321

64. Iwatsuki S, Dvorchik I, Madariaga JR, Marsh JW, Dodson F, Bonham AC et al (1999) Hepatic resection for metastatic colorectal adenocarcinoma: a proposal of a prognostic scoring system. J Am Coll Surg 189:291-299

65. Malik HZ, Prasad KR, Halazun KJ, Aldoori A, Al-Mukhtar A, Gomez D et al (2007) Preoperative prognostic score for predicting survival after hepatic resection for colorectal liver metastases. Ann Surg 246:806-814

66. Nordlinger B, Guiguet M, Vaillant JC, Balladur P, Boudjema K, Bachellier P et al (1996) Surgical resection of colorectal carcinoma metastases to the liver. A prognostic scoring system to improve case selection, based on 1568 patients: Association Française de Chirurgie. Cancer 77:1254-1262

67. Rees M, Tekkis PP, Welsh FK, O'Rourke T, John TG (2008) Evaluation of long-term survival after hepatic resection for metastatic colorectal cancer: a multifactorial model of 929 patients. Ann Surg 247:125-135

68. Zakaria S, Donohue JH, Que FG, Farnell MB, Schleck CD, Ilstrup DM et al (2007) Hepatic resection for colorectal metastases: value for risk scoring systems? Ann Surg 246:183-191

69. Leitch EF, Chakrabarti M, Crozier JE, McKee RF, Anderson JH, Horgan PG et al (2007) Comparison of the prognostic value of selected markers of the systemic inflammatory response in patients with colorectal cancer. Br J Cancer 97:1266-1270

70. McShane LM, Altman DG, Sauerbrei W, Taube SE, Gion M, Clark GM (2005) Reporting recommendations for tumor marker prognostic studies (REMARK). J Natl Cancer Inst 97:1180-1184

71. Ohtani H (2007) Focus on TILs: prognostic significance of tumor infiltrating lymphocytes in human colorectal cancer. Cancer Immun 7:4

72. Vogelstein B, Fearon ER, Hamilton SR, Kern SE, Preisinger AC, Leppert $\mathrm{M}$ et al (1988) Genetic alterations during colorectal-tumor development. N Engl J Med 319:525-532
73. Zlobec I, Steele R, Terracciano L, Jass JR, Lugli A (2007) Selecting immunohistochemical cut-off scores for novel biomarkers of progression and survival in colorectal cancer. J Clin Pathol 60:1112-1116

74. Rubbia-Brandt L, Giostra E, Brezault C, Roth AD, Andres A, Audard $V$ et al (2007) Importance of histological tumor response assessment in predicting the outcome in patients with colorectal liver metastases treated with neo-adjuvant chemotherapy followed by liver surgery. Ann Oncol 18:299-304

75. Ryan P, Nanji S, Pollett A, Moore M, Moulton CA, Gallinger S et al (2010) Chemotherapy-induced liver injury in metastatic colorectal cancer: semiquantitative histologic analysis of 334 resected liver specimens shows that vascular injury but not steatohepatitis is associated with preoperative chemotherapy. Am J Surg Pathol 34:784791

76. Fernandez FG, Ritter J, Goodwin JW, Linehan DC, Hawkins WG, Strasberg SM (2005) Effect of steatohepatitis associated with irinotecan or oxaliplatin pretreatment on resectability of hepatic colorectal metastases. J Am Coll Surg 200:845-853

77. Vauthey JN, Pawlik TM, Ribero D, Wu TT, Zorzi D, Hoff PM et al (2006) Chemotherapy regimen predicts steatohepatitis and an increase in 90-day mortality after surgery for hepatic colorectal metastases. J Clin Oncol 24:2065-2072

78. Aloia T, Sebagh M, Plasse M, Karam V, Levi F, Giacchetti S et al (2006) Liver histology and surgical outcomes after preoperative chemotherapy with fluorouracil plus oxaliplatin in colorectal cancer liver metastases. J Clin Oncol 24:4983-4990

79. Karoui M, Penna C, Amin-Hashem M, Mitry E, Benoist S, Franc B et al (2006) Influence of preoperative chemotherapy on the risk of major hepatectomy for colorectal liver metastases. Ann Surg 243:1-7

80. Klinger M, Eipeldauer S, Hacker S, Herberger B, Tamandl D, Dorfmeister M et al (2009) Bevacizumab protects against sinusoidal obstruction syndrome and does not increase response rate in neoadjuvant XELOX/FOLFOX therapy of colorectal cancer liver metastases. Eur J Surg Oncol 35:515-520

81. Aussilhou B, Dokmak S, Faivre S, Paradis V, Vilgrain V, Belghiti J (2009) Preoperative liver hypertrophy induced by portal flow occlusion before major hepatic resection for colorectal metastases can be impaired by bevacizumab. Ann Surg Oncol 16:1553-1559

82. Saif MW (2009) Secondary hepatic resection as a therapeutic goal in advanced colorectal cancer. World J Gastroenterol 15:3855-3864

83. Van Cutsem E, Rivera F, Berry S, Kretzschmar A, Michael M, DiBartolomeo M et al (2009) Safety and efficacy of first-line bevacizumab with FOLFOX, XELOX, FOLFIRI and fluoropyrimidines in metastatic colorectal cancer: the BEAT study. Ann Oncol 20:1842-1847

84. Dindo D, Demartines N, Clavien PA (2004) Classification of surgical complications: a new proposal with evaluation in a cohort of 6336 patients and results of a survey. Ann Surg 240:205-213

85. Chong G, Cunningham D (2005) Improving long-term outcomes for patients with liver metastases from colorectal cancer. J Clin Oncol 23: 9063-9066

86. Gruenberger B, Tamandl D, Schueller J, Scheithauer W, Zielinski C, Herbst F et al (2008) Bevacizumab, capecitabine, and oxaliplatin as neoadjuvant therapy for patients with potentially curable metastatic colorectal cancer. J Clin Oncol 26:1830-1835

87. National Comprehensive Cancer Network. Colon cancer practice guidelines in oncology. Available from: http://www.nccn.org

88. Hicklin DJ, Ellis LM (2005) Role of the vascular endothelial growth factor pathway in tumor growth and angiogenesis. J Clin Oncol 23: 1011-1027

89. Ichante JL, Adenis A, Francois E, Boucher E, Pignon JR, Ychou M et al (2011) Impact of early tumour shrinkage on long-term outcome in metastatic colorectal cancer ( $\mathrm{mCRC}$ ) treated with $5 \mathrm{FU}+$ irinotecan + leucovorin (FOLFIRI) or capecitabine + irinotecan (XELIRI) plus bevacizumab. Eur J Cancer 47:S419 